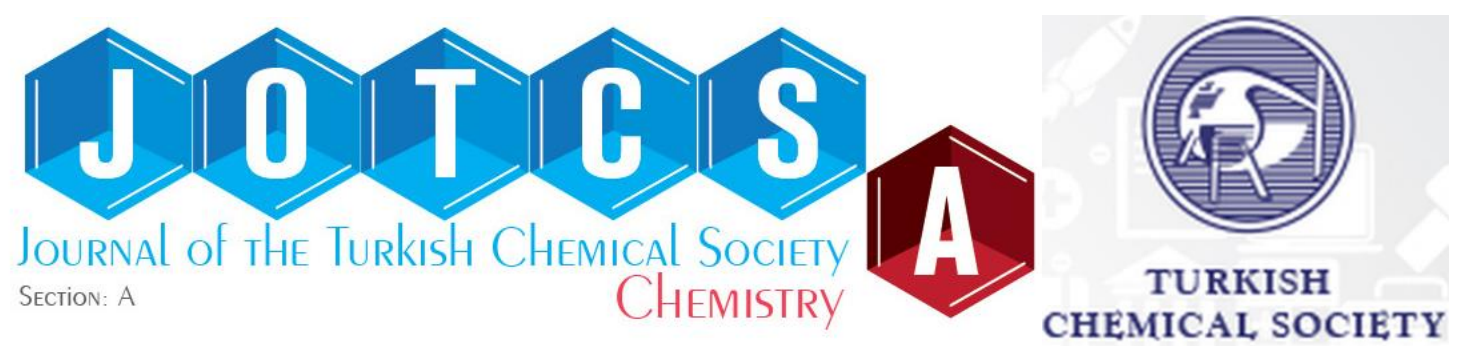

\title{
Facile Synthesis of Semiconducting Nanosized OD and 2D Lead Oxides Using a Modified Co-Precipitation Method
}

\author{
Ahmet Güngör ${ }^{(D)}$, Rükan Genç ${ }^{1,2 *}$, Tonguç Özdemir ${ }^{1}(i)$ \\ ${ }^{1}$ Department of Chemical Engineering, Mersin University, Mersin, Turkey, 33343 Mersin, Turkey \\ ${ }^{2}$ Advanced Technology Research and Application Center, Mersin University, 33343, Mersin, \\ Turkey.
}

\begin{abstract}
Nano-sized lead has many versatile applications that could be useful in daily life. In the present work, we report a comprehensive study for preparation of nanosized lead oxide using the co-precipitation method and optimization of reaction parameters to obtain lead oxide ( $\mathrm{PbO})$ nanoparticles with homogeneously distributed size, shape, and structure. When aqueous solution of lead(II) acetate was reacted with sodium hydroxide at elevated temperatures, the alpha form of lead oxide nanoparticles, with spherical shape were produced. Decreasing the ratio of sodium hydroxide to lead(II) acetate concentration at moderate temperatures resulted in a gradual change in crystal structure from quasi-spherical a-PbO nanoparticles and two dimensional nanoflakes of beta $\mathrm{PbO}$ with a thickness below $100 \mathrm{~nm}$ was synthesized for the first time. The particles obtained in both $a$ and $\beta$ forms were characterized by $X$-ray powder diffraction (XRD), dynamic light scattering equipment (Zetasizer), transmission electron microscopy (TEM), scanning electron microscopy (SEM) and Fourier transform infrared spectroscopy (FTIR) techniques. Finally, thin layers of freeze-dried $\mathrm{a}-\mathrm{PbO}$ and $\beta-\mathrm{PbO}$ particle powder on glass and filter paper were formed by the help of nail polisher and conductivity measurements were performed using four-point probes method. Produced layers $\beta$ - $\mathrm{PbO}$ particles of the size below $100 \mathrm{~nm}$ showed higher conductivity on both supports as compared to the ones produced from spherical a-PbO nanoparticles. This altered the conductivity of the material in the semiconducting zone, which is probably due to a more effective electron transfer facilitated by 2D alignment of the molecules, could increase the potential use of this material in voltaic and catalysis.
\end{abstract}

Keywords: Lead oxide nanoparticle, 2D lead oxide, co-precipitation, nanomaterials, semiconducting thin layer.

Submitted: July 18, 2017. Accepted: September 21, 2017.

Cite this: Güngör A, Genç R, Özdemir T. Facile Synthesis of Semiconducting Nanosized OD and 2D Lead Oxides Using a Modified Co-Precipitation Method. JOTSA. 2017;4(3):1017-30.

DOI: $10.18596 /$ jotcsa. 329272 .

*Corresponding author. E-mail: rukangnc@gmail.com. 


\section{INTRODUCTION}

Enhanced properties of the matter such as low-density, high surface area to volume ratio, structure depended physical properties and so on as the size is reduced to nano-level caused an increase in the number of studies towards understanding the structural characteristics of different materials or developing new methods or improving the existing ones in order to achieve a bulk synthesis of nanoparticles with controllable size, shape and function (1-3). Up until now, various synthesis methods including electrolysis, sonochemical synthesis (4), co-precipitation (5), hydrothermal (6), thermal decomposition (7), and spray pyrolysis (8) have been developed for nanomaterial synthesis and they were applied to expand the number of chemical compositions including soft-materials $(9,10)$, metals/metal oxides (11-14) and/or their composites (15-17). However, for each formulation, re-optimization is required since the final molecular organization and size of the nanoparticles formed as a result are defined by supramolecular interactions of individual molecules, and therefore synthesis parameters should be specified accordingly.

Lead and its compounds have been utilized in various areas for many centuries. Lead oxide can be found in many crystalline forms such as $\mathrm{a}$ and $\beta \mathrm{PbO}, \mathrm{PbO}_{2}$, and so on (18). Lead(II) oxide is a member of lead(II) binary oxides and has two different crystal structures, a-PbO, litharge (tetragonal form), and $\beta-\mathrm{PbO}$, massicot (orthorhombic form) which are known as photoactive semiconductor materials $(19,20)$. Particularly, nanosized lead oxide has attracted the attention of researchers in recent years for the wide range of applications as passivation layer (21), as sulfide sensor (22), lead acid battery (23), which in parallel bring out the need for methods of controlled synthesis of nano-sized lead oxides and lead dioxides, however, literature on the synthesis procedures for controlled synthesis of lead-based nanomaterials are still limited.

Wilkinson et al. (20) have reported the synthesis of tetragonal alpha-lead oxide nanoparticle using a simple co-precipitation method. In another original approach, Hanifehpour et al. (24) have used two-step thermal decomposition method for the synthesis of alpha-lead oxide nanoparticles. For this purpose, firstly they have synthesized 1D polymeric lead(II) complex by the sonochemical method, and a further thermolysis of $1 \mathrm{D}$ polymeric lead(II) complex at $180^{\circ} \mathrm{C}$ resulted in $\mathrm{PbO}$ nanoparticles of $33 \mathrm{~nm}$. Using the same approach with few modifications, Hashemi et al. (14) also synthesized lead(II) complex with polymer formed via sonochemical method and reached the particle size of around $95 \mathrm{~nm}$. Zhou et al. (25) have studied the synthesis of nano-sized alpha and beta form $\mathrm{PbO}$ particles by decomposition of lead citrate which were then successfully implemented to lead-acid battery paste as a starting material. They have showed that particle size and crystal structure is defined by the presence of oxygen in the synthesis media Ranjbar et al. (26) have studied the synthesis of $\mathrm{Pb}$ (II) coordination polymer by a sonochemical method and they obtained finally the nanomaterial as pure alpha-lead oxide (a-PbO) with an average particle size of $70 \mathrm{~nm}$. Recently, Shin et al. (27), have synthesized 
nanoflower lead(II) coordination compound with a mean size of $42 \mathrm{~nm}$ using the sonochemical method. After decomposition of this compound at $180{ }^{\circ} \mathrm{C}$ in the presence of oleic acid, $\mathrm{PbO}$ nanoparticles were obtained. SEM and TEM analyses showed that the size of obtained PbO was about $35 \mathrm{~nm}$. As far as being known, the literature on the nanosized lead oxide is limited to these research reports and there is still a plenty of room for new and sophisticated methods of preparation leading to nanoparticles or structures, especially for the synthesis of highly active lead-based nanostructures organized in 2D nanostructures.

In this study, we reported a detailed investigation on the lead oxide nanoparticle synthesis and characterization. We optimized the co-precipitation method (20) by evaluating several experimental parameters, such as temperature, lead(II) acetate (Pb-Ac) and sodium hydroxide concentrations, performing a purpose-designed experiment. Particle characterization was done by powder XRD, Zetasizer, FE-SEM, TEM, and FTIR. Formation of alpha and beta lead oxide nanostructures ordered in $\mathrm{OD}$ and $2 \mathrm{D}$ and their semiconducting thin films were reported.

\section{MATERIALS AND METHODS}

\section{Materials}

Bulk lead(II) acetate trihydrate $\left(\mathrm{Pb}\left(\mathrm{C}_{2} \mathrm{H}_{3} \mathrm{O}_{2}\right)_{2} \cdot 3 \mathrm{H}_{2} \mathrm{O}\right)$ powder, sodium hydroxide $(\mathrm{NaOH})$, and acetic acid $\left(\mathrm{CH}_{3} \mathrm{COOH}\right)$ were obtained from Sigma-Aldrich. All chemicals were used as received.

\section{Preparation of lead(II) oxide nanoparticles}

As illustrated in Figure 1, previously heated $\left(90^{\circ} \mathrm{C}\right)$ aqueous solution of $1.2 \mathrm{M}$ lead(II) acetate trihydrate in de-ionized water $(60 \mathrm{~mL})$ was directly poured into $50 \mathrm{~mL}$ of $19 \mathrm{M} \mathrm{NaOH}$ in a glass beaker under vigorous mixing. Once the solution's color turned to red, mixing was stopped and the sediments were allowed to settle. The reaction was carried out in a sonic bath rather than using the magnetic stirring that was used in previously reported studies (20) in order to achieve a better size distribution. By this way, we expected to transfer homogenously distributed kinetic energy to the molecules in order to prevent particle agglomeration during nucleation. Once the color change from white to red (alpha form) or white to green (beta form) was seen, the reaction was stopped and the supernatant liquid was discharged. The sediment was filtered on a Buchner funnel and then the precipitate was washed with de-ionized water. In standard co-precipitation method (20), the particles are dried in an oven and agglomerated particles are crushed in a mortar in order to obtain a homogenous powder of reduced the size. However, we concluded that this step is unnecessary since it caused the loss of nanoparticles and leads to the formation of large particles to agglomerate. For this reason, we rather did the drying procedure with a lyophilization step suitable for aqueous solutions. To do so, we placed the liquid solution of nanoparticles in a freeze-dryer at $-80^{\circ} \mathrm{C}$ overnight. The frozen particles were then located in a low-temperature freeze-dryer and dried again through lyophilization overnight. The obtained powder was stored in a container. The so-synthesized particles were kept for further analysis. 


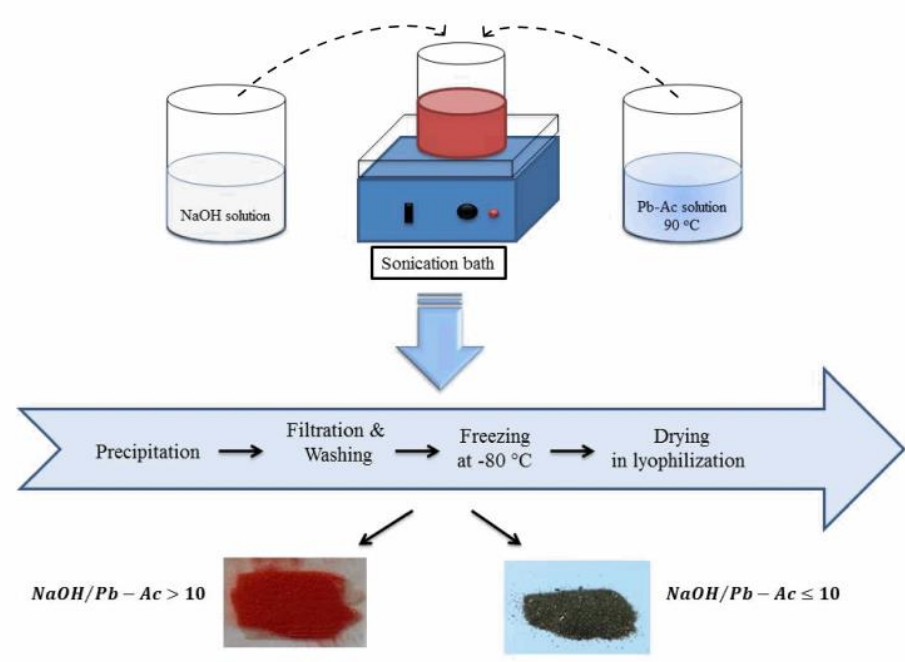

Figure 1: Schematic illustration of synthesis and modification process.

\section{Characterization methods}

Scanning Electron Microscopy Imaging: Particle morphology of $\mathrm{PbO}$ particles were observed by field emission scanning electron microscopy (FE-SEM) using a Zeiss Supra 55 instrument at 10 $\mathrm{kV}$ with magnification of 1000 to 100.000 . Particle size distribution of spherical nanoparticles (a$\mathrm{PbO}$ ) and mean thickness of the $2 \mathrm{D}$ nanostructures $(\beta-\mathrm{PbO})$ were calculated using Image $]$ analysis of the SEM images. The mean diameter was calculated by measuring either diameter or the thickness of over 50 nanostructures. The dimensions of the nanoparticles were calculated by measuring the size of each particle in the SEM images using the "J image" program and taking the averages.

$X$-ray diffraction analysis: Crystal structures and particle size were determined by the use of powder X-ray diffraction (XRD) using a Rigaku Smartlab powder diffractometer with $\mathrm{Cu}$-Ka radiation at $2 \theta$ between $10^{\circ}-90^{\circ}$. Williamson-Hall and Debye-Scherer methods were used for the determination of the particle size of $O D(a-P b O)$ nanostructure $(28,13)$. Data gathered from XRD analysis were used to calculate the average grain size of alpha lead oxide nanoparticles via Scherer method (Equation 1) where $\lambda=0.1541 \mathrm{~nm}, " \beta$ " is full width at half maximum (FWHM), " $\theta$ " is the diffraction angle and ' $D$ ' is particle size diameter.

$$
D=\frac{k \lambda}{\beta \cos (\theta)}
$$

Alpha lead oxide particle size can also be calculated from Williamson-Hall plot using Equation 2.

$$
\beta * \cos (\theta)=\frac{k \lambda}{D}+4 \varepsilon \sin (\theta)
$$

Using the XRD analysis data, appropriate graphs were plotted. From these plots, "k $\lambda / D^{\prime}$ values (where $\mathrm{k}$ is a constant variable number depending on the crystal structure, which is 0.9 for $\mathrm{PbO}$ 
particle size analysis: $\lambda$ is wavelength; $D$ is particle size) were calculated. By this way, particle size distribution of each sample was compared with the results obtained from SEM, TEM, and Zetasizer.

Transmission Electron Microscopy Analysis: In the study, characterization of the particle morphology and the size of the samples was carried out using a JEOL JEM 1011 TEM apparatus. Dried particles were immersed in $10 \%$ ( $v: \mathrm{v}$ ) acetic acid solution and a drop of this sample was deposited on a $\mathrm{Cu}$ microgrid and dried at room temperature. Particle morphology and size analysis were performed at $80 \mathrm{keV}$ with an ultrahigh-resolution pole piece providing a point resolution of $2 \AA$. Images were analyzed with an TEM image analysis platform and particle size distribution was plotted by measuring the radius of at least 100 particles.

Particle Size Distribution via Dynamic Light Scattering (DLS): The mean diameter of prepared alpha lead oxides and size distribution through the sample were measured through Zetasizer Nano ZS from Malvern Instruments, Inc., at $25 \pm 0.5{ }^{\circ} \mathrm{C}$ (HeNe laser $(633 \mathrm{~nm})$, detector angle of 173 degrees), which measures the rate of undulation of the light scattered from the particles. Standard deviation calculated from the mean diameter of samples was prepared in triplicate. The $10 \%(v: v)$ acetic acid solution was used as a solvent.

Fourier Transform Infrared Spectroscopy Analysis: Frontier model Perkin Elmer FTIR Spectrometer was used to characterize the vibrational modes and transmittance values. The FTIR spectra were recorded in the range $400-4000 \mathrm{~cm}^{-1}$.

Four-point probe conductivity measurement: Thin layers of both alpha and beta lead oxide nanostructures were obtained by embedding nanoparticle powders on glass slides and cellulose membrane $(0.5 \times 0.5 \mathrm{~cm})$ pre-treated with a layer of nail polish (100 mg nanoparticle in $1 \mathrm{~mL}$ ). The conductivity of dried layers was further measured using Entek Brand four-point probe electrodes.

\section{RESULTS AND DISCUSSION}

\section{Synthesis of a-PbO nanoparticles and characterization}

The obtained particles were analyzed by XRD in order to determine the crystal structure. As shown in Figure 2, particles prepared with original conditions (20) but in a sonic bath and dried with lyophilization showed X-ray diffraction pattern identified as pure alpha-lead oxide (PDF card no: $01-078-1665)$. The major peaks located at $17.64^{\circ}, 28.62^{\circ}, 31.82^{\circ}, 35.72^{\circ}$, indexed as (001), (111), (020) and (002) planes, respectively, are characteristic peaks for the pure tetragonal phase of a-PbO nanoparticles (29). 


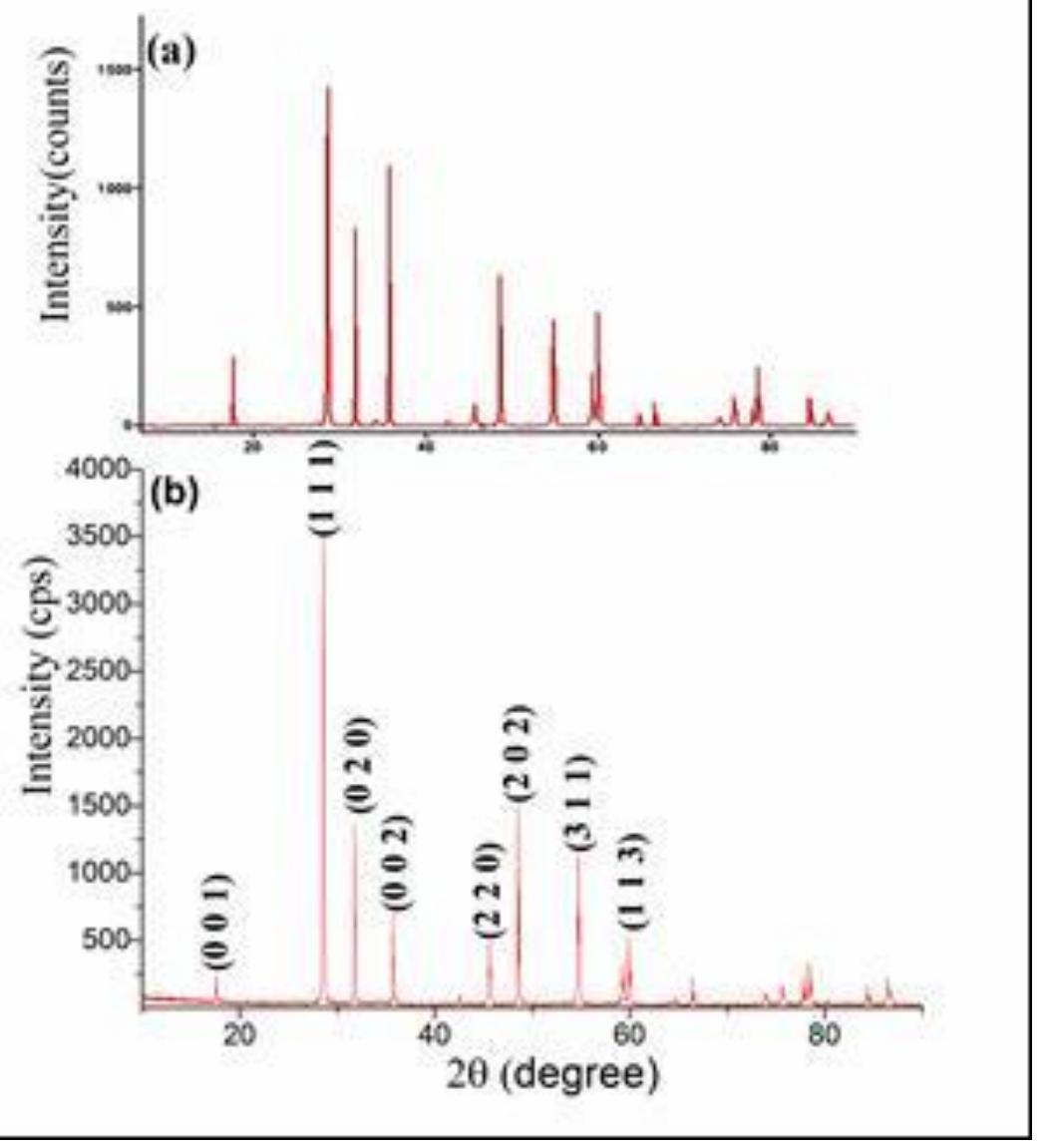

Figure 2: XRD patterns of (a) pure a-PbO, (b) synthesized lead oxide particles.

As mentioned before, intercept value in the Williamson-Hall Method and slope value in the Debye-Scherer method were used to calculate the " $k \lambda / D$ " values. Mean particle sizes of $a-P b O$ were calculated as $38.5 \mathrm{~nm}$ and $36.4 \mathrm{~nm}$ from intercept and slope values, respectively.

Particle size of alpha lead oxide (a-PbO) in acetic acid ( $10 \% \mathrm{v}: \mathrm{v})$ was measured using Zetasizer Nano Series and size distribution diagrams given in Figure 3. As depicted in Figure 3, when particles were prepared with a $19 \mathrm{M} \mathrm{NaOH}$ and $1.2 \mathrm{M} \mathrm{Pb}-\mathrm{Ac}$ at constant solution temperature of $90{ }^{\circ} \mathrm{C}$, the mean hydrodynamic diameter for the particles were found to be $30.91 \pm 6.1 \mathrm{~nm}$. These results show very close match to the values obtained from XRD measurements.

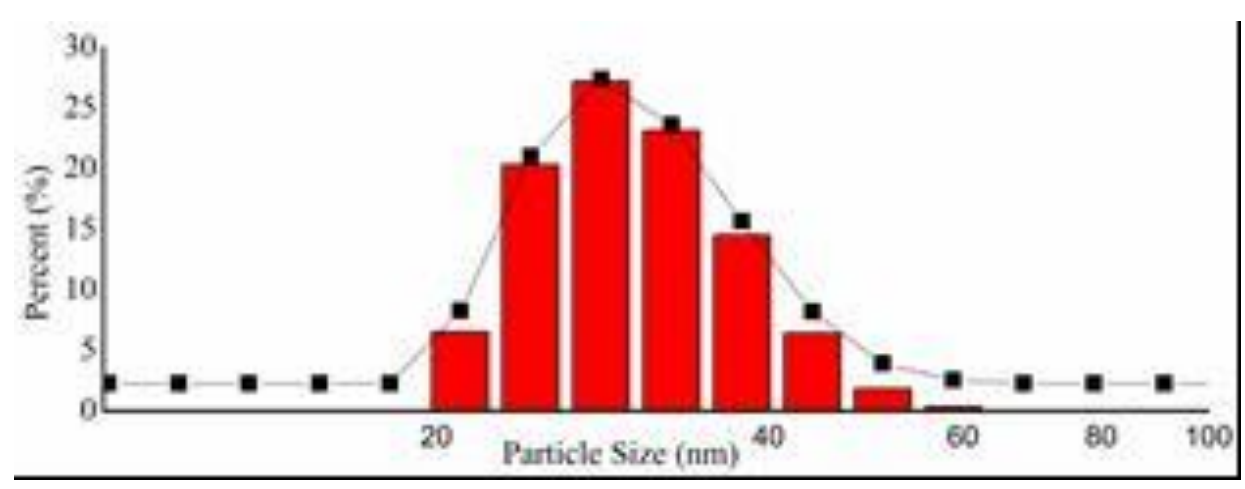

Figure 3: Particle size distribution of $\mathrm{PbO}$ with DLS measurement. 


\section{The effect of different parameters on particle size and structure}

In order to have a controllable final particle structure and homogeneity in size, optimization studies were conducted by changing important experimental parameters that play effective role in particle growth. For this purpose, the effect of three parameters namely (1) the concentration of sodium hydroxide and (2) lead(II) acetate and (3) lead(II) acetate solution temperature on the final nanoparticle size and the shape were explicitly evaluated and the results were given in Figure 4.

a) The effect of $\mathrm{NaOH}$ concentration on particle size: It is well known that the initial concentration of precipitating agent, $\left[\mathrm{OH}^{-}\right]$ion, effects the nucleation and growth rate of nanoparticles and thus it is effective on the final molecular structure of the particles (30). Herein, sodium hydroxide concentration was used in the range of $10 \mathrm{M}$ to $19 \mathrm{M}$ and the intrinsic effect of these parameters on the final nanoparticle characteristics were examined at constant lead(II) acetate concentration of $1.2 \mathrm{M}$ at $90^{\circ} \mathrm{C}$. As shown in Figure $4(\mathrm{a})$, PbO nanoparticle size decreased with the increased $\mathrm{NaOH}$ concentration from 10 to $14.5 \mathrm{M}$ implying an improved nucleation rate that limits the growth of larger particles $(28,31)$. However, when $\mathrm{NaOH}$ concentration was further increased to $19 \mathrm{M}$, an increase in nanoparticle size was observed probably due to the fast reduction of the lead molecules at elevated concentrations of $\mathrm{NaOH}$ which initiates a molecular aggregation of larger sized particles. The data shown in Figure 3 suggest that the sodium hydroxide species at $\mathrm{NaOH}$ concentration of $14.5 \mathrm{M}$ reconstruct the lead oxide surface to generate domains with the smallest dimension of $27.48 \pm 5.6 \mathrm{~nm}$ and narrowest particle size distribution (32).

\section{b) Pb-Ac concentration vs. Particle size}

In order to understand the effect of the lead(II) acetate concentration on the particle size, the lead(II) acetate concentration was varied between 0.8 to $1.2 \mathrm{M}$ while keeping the sodium hydroxide concentration at $14.5 \mathrm{M}$ and initial lead solution temperature at $90{ }^{\circ} \mathrm{C}$. As shown in Figure 4(b), PbO nanoparticles' size increased with the increased Pb-Ac concentration. In this case, smallest particle size $(17.72 \pm 5.6 \mathrm{~nm})$ was obtained when lead(II) acetate solution concentration was kept at $0.8 \mathrm{M}$ which leads us to a certain $\mathrm{NaOH} / \mathrm{lead}$ (II) acetate ratio of 18.125. When this ratio was increased, available precursor number per reducer increases, and as a result, a fast accumulation over the seeds occurs and gives rise to larger agglomerates as we observed in the case of elevated $\mathrm{NaOH}$ concentrations $(23,33)$. 

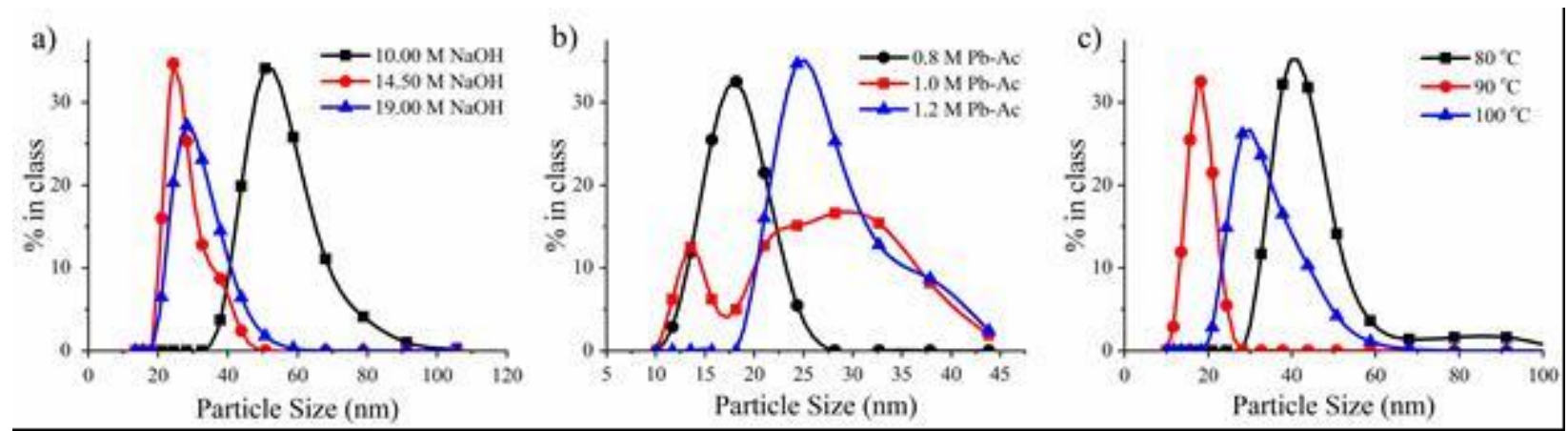

Figure 4: Size distribution graphs at different concentrations of (a) $\mathrm{NaOH},(b) \mathrm{Pb}-\mathrm{Ac}$ and (c) at a number of operation temperatures for $\mathrm{Pb}-\mathrm{Ac}$.

c) The effect of $P b-A c$ solution temperature on particle size

In addition to the reactant concentrations and their ratio affecting the molecular accumulation and crystallization of the molecules into different micro/nano structures, the solution temperature has also a major role in particle synthesis by directly affecting the reaction kinetics. In order to understand the effect of temperature on the particle size, the lead(II) acetate solution's temperature was varied between 80 to $100{ }^{\circ} \mathrm{C}$ while keeping sodium hydroxide and lead(II) acetate concentrations at 14.5 $\mathrm{M}$ and $0.8 \mathrm{M}$, respectively. As depicted in Figure 4(c), the smallest lead oxide particles were observed at $90^{\circ} \mathrm{C}$. The particles obtained under optimum conditions were analyzed by XRD, SEM and TEM. XRD crystal structure analysis result is shown in Figure 5. The XRD spectra of these particles showed that the characteristic diffraction peaks illustrating the presence of lead oxide in pure alpha form. However, at the lead(II) acetate solution temperature beyond $90^{\circ} \mathrm{C}$, agglomeration occurs due to the increased reaction rates (34). These results clearly showed that supramolecular and intermolecular interactions of the lead molecules are notably affected by the operation temperature and at temperatures higher than $90^{\circ} \mathrm{C}$, particles for large aggregates rather than tiny nanoparticles becomes significant (Figure 6).

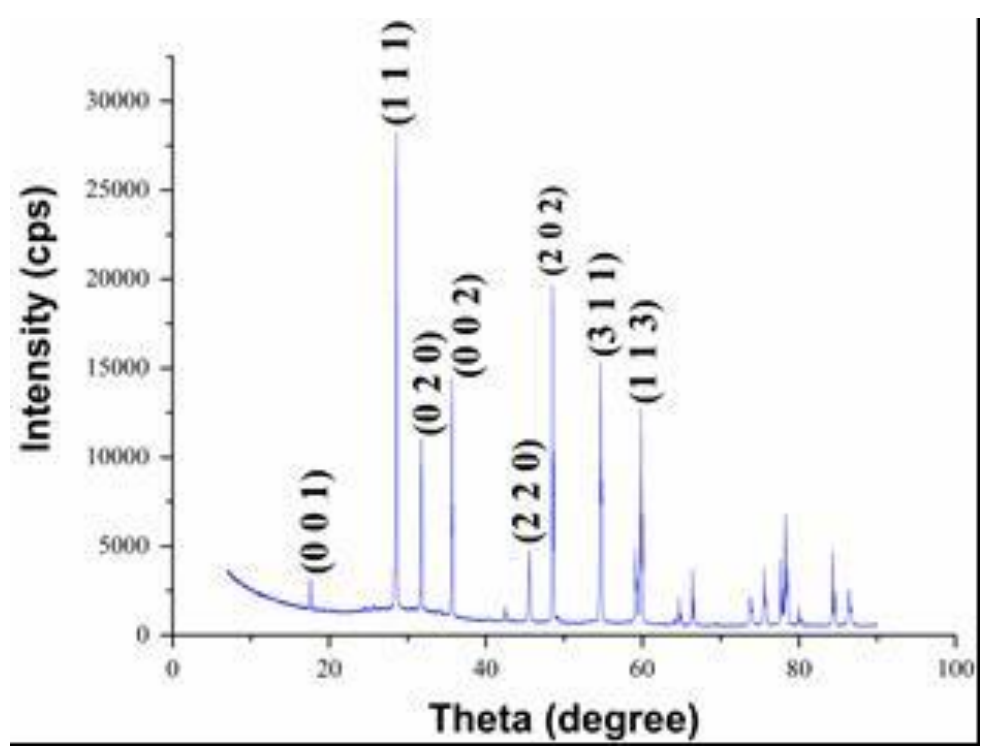

Figure 5: XRD patterns of synthesized lead oxide particles under optimal conditions. 
The results of the optimization studies revealed that $O D$ PbO nanoparticles of $17.72 \pm 5.6 \mathrm{~nm}$, which are quite smaller than nanoparticles synthesized with the certain methods in the literature, can be achieved by applying co-precipitation method involving a simple recipe that include a mixture of aqueous solutions of $14.5 \mathrm{M}$ sodium hydroxide as reactant and $0.8 \mathrm{M}$ lead acetate pre-heated to $90^{\circ} \mathrm{C}$ as starting processor (Figure 1).

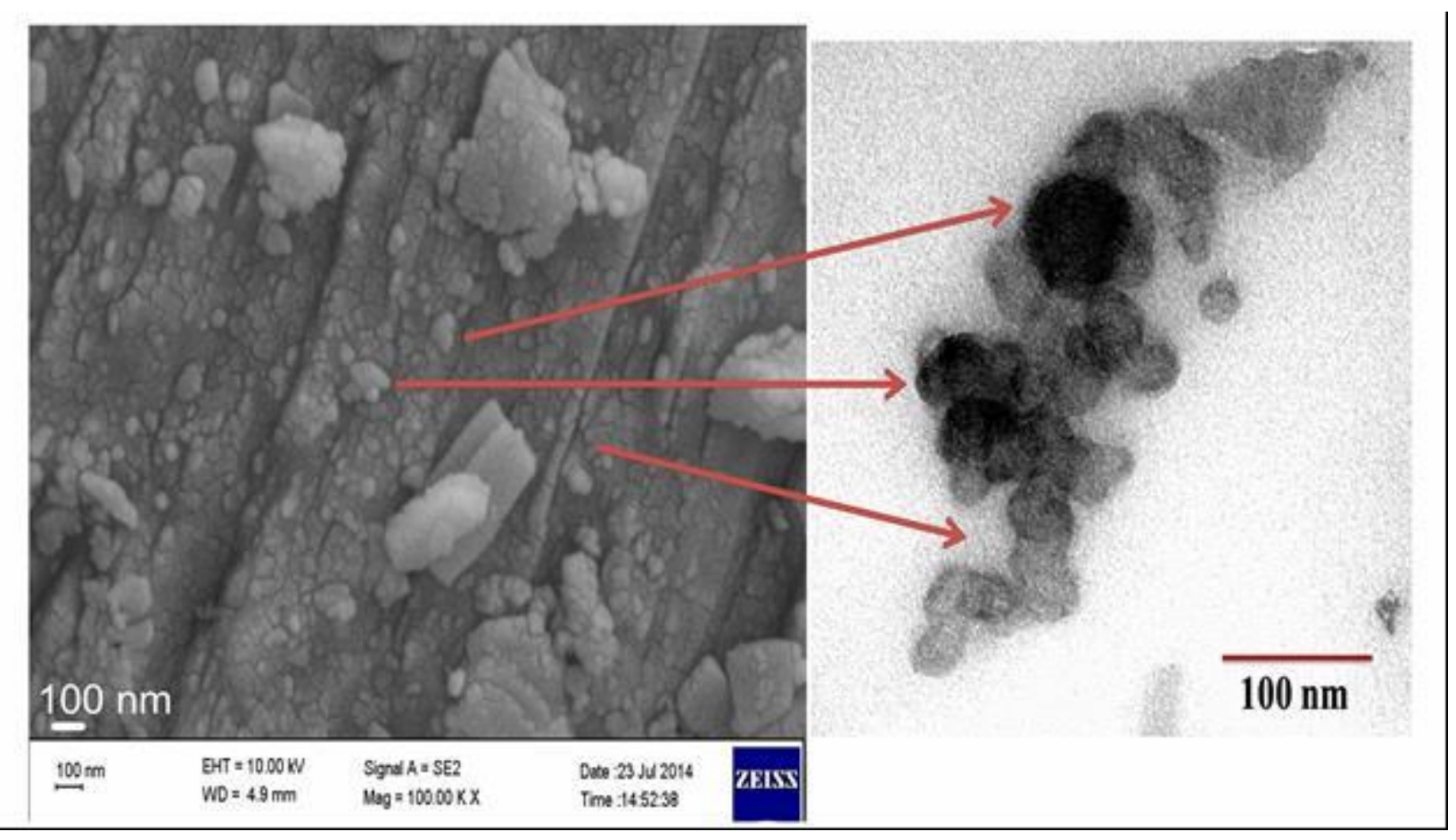

Figure 6: SEM and TEM images of a-PbO particles.

\section{Synthesis of $\boldsymbol{\beta}-\mathrm{PbO}$ nanoparticles}

In optimization studies, when the $\mathrm{NaOH} / \mathrm{Pb}-\mathrm{Ac}$ molar ratio was decreased to a certain value, it was seen that when $\mathrm{NaOH} / \mathrm{Pb}-\mathrm{Ac}$ molar ratio equals or below 10 , a green and shiny sample color appears instead of the regular reddish colored a-PbO particles obtained at higher $\mathrm{NaOH} / \mathrm{Pb}-\mathrm{Ac}$ ratios. Table 1 summarizes the optimization studies at $90^{\circ} \mathrm{C}$ showing the synergetic effect of both the concentrations of $\mathrm{NaOH}$ and $\mathrm{Pb}-\mathrm{Ac}$ in the form of $\mathrm{NaOH} /(\mathrm{Pb}-\mathrm{Ac})$ on the final crystal structure.

Table 1: Reaction parameters and their effect on the final crystal structure of lead oxides.

\begin{tabular}{|c|c|c|c|c|c|c|c|c|c|}
\hline \multirow[b]{3}{*}{$\begin{array}{l}\text { Pb-Ac Conc. } \\
\text { (mole/L) }\end{array}$} & \multicolumn{9}{|c|}{$\mathrm{NaOH}$ Concentration $(\mathrm{mol} / \mathrm{L})$} \\
\hline & \multicolumn{3}{|c|}{$10 \mathrm{M} \mathrm{NaOH}$} & \multicolumn{3}{|c|}{$14,5 \mathrm{M} \mathrm{NaOH}$} & \multicolumn{3}{|c|}{$19 \mathrm{M} \mathrm{NaOH}$} \\
\hline & 0,8 & 1 & 1,2 & 0,8 & 1 & 1,2 & 0,8 & 1 & 1,2 \\
\hline $\begin{array}{c}\mathrm{NaOH} / \mathrm{Pb}-\mathrm{Ac} \\
\text { Ratio }\end{array}$ & 12,5 & 10 & 8,33 & 18,12 & 14,5 & 12,08 & 23,75 & 19 & 15,83 \\
\hline Crystal Structure & $\mathrm{a}-\mathrm{PbO}$ & $\beta-\mathrm{PbO}$ & $\beta-\mathrm{PbO}$ & $\mathrm{a}-\mathrm{PbO}$ & $\mathrm{a}-\mathrm{PbO}$ & $\mathrm{a}-\mathrm{PbO}$ & $\mathrm{a}-\mathrm{PbO}$ & $\mathrm{a}-\mathrm{PbO}$ & $\mathrm{a}-\mathrm{PbO}$ \\
\hline
\end{tabular}


The crystal structure of synthesized green and shiny particles chart was shown in Figure 7 where it can clearly be seen that the peaks mainly overlapped with the $\beta$-PbO structure (PDF card no: 00-005-0570) but some of the peaks correspond to a-PbO. Consequently, the synthesized green particles are substantially in the form of $\beta-\mathrm{PbO}$.

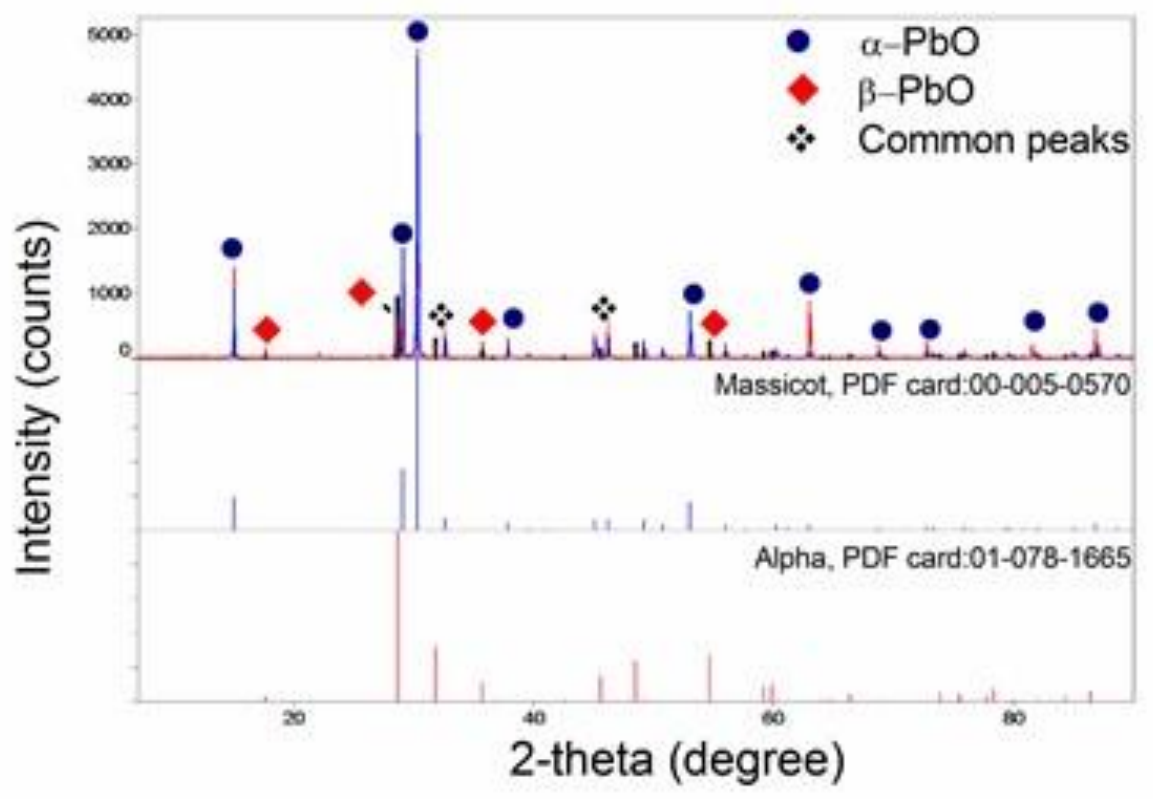

Figure 7: XRD patterns of synthesized $\beta-\mathrm{PbO}$ particles.

Electron microscopic images of the lyophilized sample in powder form depicted in Figure 8 showing that the smaller sodium hydroxide to lead(II) acetate molar ratio leads to the formation of 2D PbO nanoflakes in beta form. The thicknesses of the nanostructures were measured by Image J program using SEM images captured. As presented in FE-SEM images, the synthesized two dimensional $\beta$-PbO particles with micron-sized width have thickness below $100 \mathrm{~nm}$ and their width ranges from tens to several hundred micrometers.

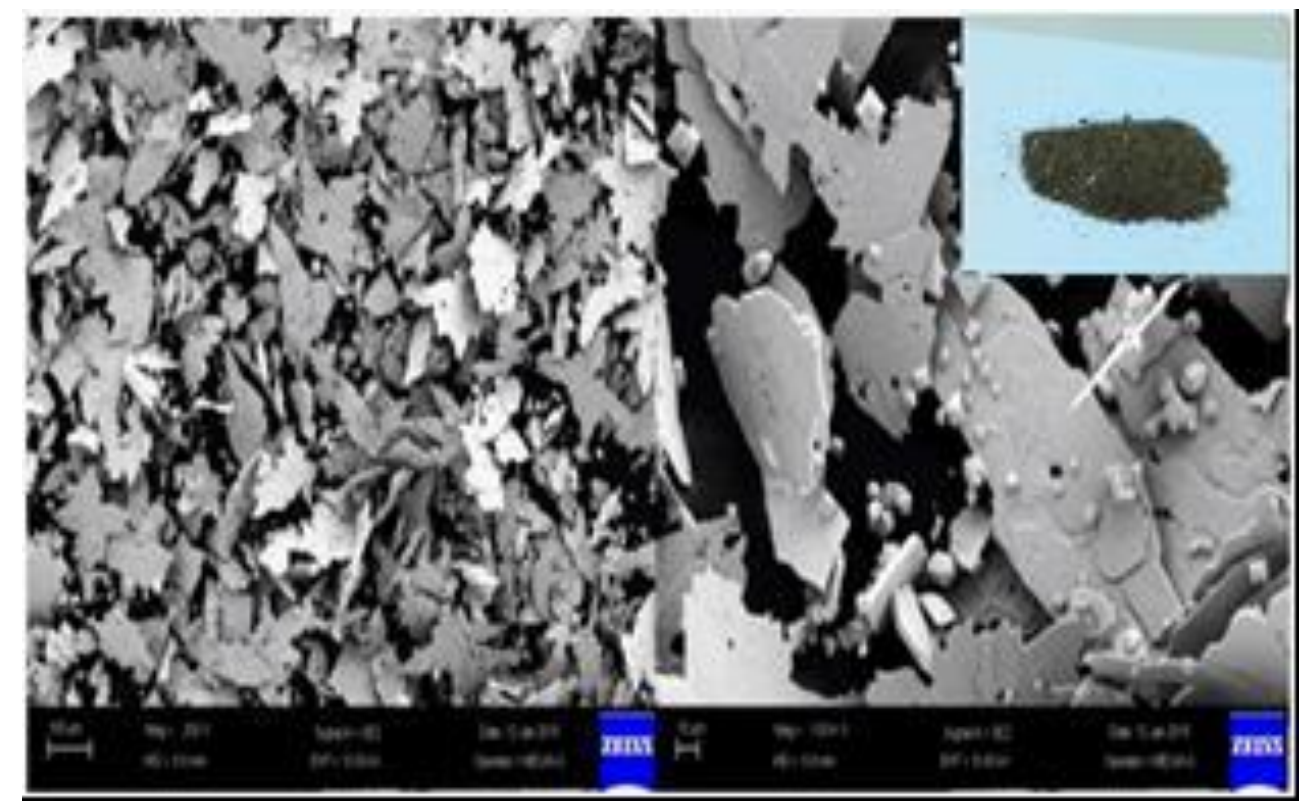

Figure 8: FE-SEM images of synthesized $\beta$-PbO particles. 


\section{FTIR analysis}

FTIR spectra for alpha and beta form of lead oxides are given in Figures 9(a) and 9(b), respectively. Both samples presented a strong peak at $1393 \mathrm{~cm}^{-1}$ corresponding to the $\mathrm{Pb}-\mathrm{O}$ stretching and another peak at $682.9 \mathrm{~cm}^{-1}$ that indicates the presence of lead (35).
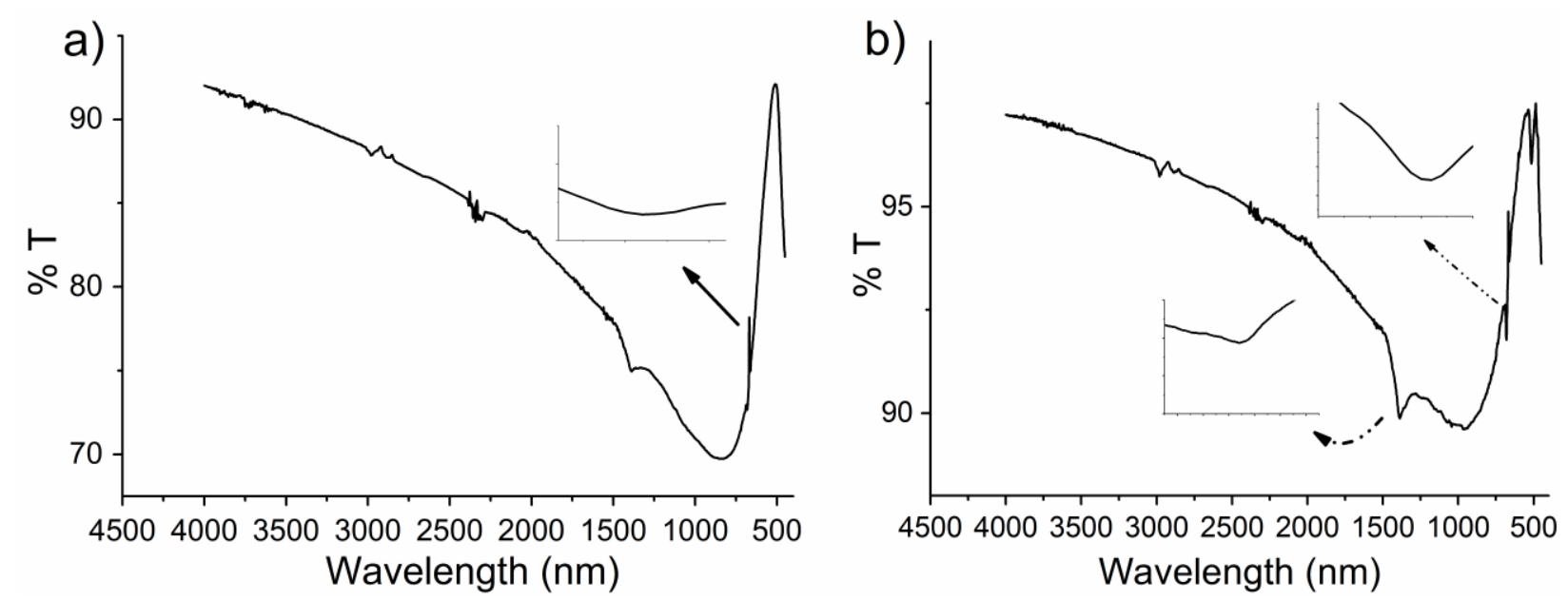

Figure 9: FTIR spectra of (a) a-PbO and (b) $\beta$-PbO nanoparticles.

\section{Conductivity Analysis}

$\mathrm{PbO}$ is known as a photoactive semiconductor with a band gap of 1.92 (19). These compounds are used as passivation layers (21), oriented lead oxide on silica and lead oxide-matrix composites (36). Lead oxides have strong absorption cross sections, high carrier mobility, and conductivity (37). We performed four-point probe analysis on the thin layers of each $\mathrm{PbO}$ nanoparticles formed on two different supports: glass slide and cellulose filter paper as a proof of concept study for their future applications of them in electrochemistry and energy issues. Thin layers were prepared by embedding particles on the support with the help of nail polisher. As seen in Figure 10, both alpha and beta form lead oxide thin layers showed conductivities values of 0.232 and $0.333 \mathrm{~S} / \mathrm{m}$ on glass and 1.92 and $4.07 \mathrm{~S} / \mathrm{m}$ on cellulose filter paper, respectively. Although, both materials have shown to be semiconducting, beta formed 2D nanoflakes showed higher conductivity than that of quasi-spherical alpha lead oxide nanoparticle layer as expected. This difference was possibly occurred due to a facilitated electron transfer by the 2D lattice structure of the material. 


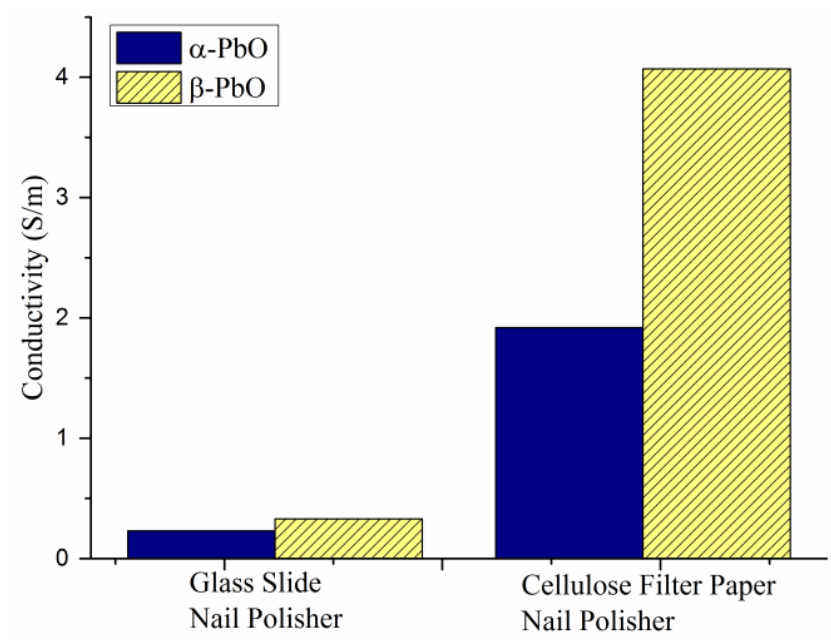

Figure 10: Conductivity of the particles synthesized on different surfaces.

\section{CONCLUSIONS}

In this study, co-precipitation method for the multi- gram scale synthesis of lead oxide ( $\mathrm{PbO}$ ) nanoparticles in different crystalline structures was optimized at different temperatures and different concentrations of lead(II) acetate and sodium hydroxide. Both the concentration of the reactants and the processing temperature synergistically have an influence on the size of the final lead oxide nanostructures. On the other hand, we concluded unusually that one can also tune the crystal structure of the lead oxide by tailoring the sodium hydroxide /lead(II) acetate molar ratio to certain values. At sodium hydroxide to lead(II) acetate molar ratios equals or less than $10, \beta-P b O$ based $2 \mathrm{D}$ nanoflakes with thicknesses below $100 \mathrm{~nm}$ and widths reaching to several micrometers were obtained for the first time in the literature and the process is temperature-independent. On the other hand, the semi-spherical nanoparticles of a-PbO with a mean size below $20 \mathrm{~nm}$ were formed at higher molar ratios. Thin layers of both alpha and beta nanostructure formations on both glass and cellulose paper were shown to be semi-conductive where 2D nanoflakes showed higher conductivity which is possibly due to easily electron transfer through the lead molecules when organized in 2D. The so-prepared nanostructures with homogenous in size and shape could have a great potential in photovoltaic studies, especially in third generation perovskite solar cells and electrochemical and catalytic applications.

\section{ACKNOWLEDGMENTS}

This study was funded by The Scientific and Technological Research Council of Turkey (TUBITAK) under the project number of 213M476. The authors would like to thank Res. Assist. İsmail Kutlugün Akbay for his valuable contributions.

\section{REFERENCES}

1. Weng L, Yan L, Li H, et al. Facile fabrication and properties of core - shell structure $\mathrm{Ag} @ \mathrm{Al} 2(\mathrm{SiO} 3) 3$ 
nanocomposites with controllable morphologies. Mater. Lett. Internet.. 2014;126:240-243.

2. Li Y, Qiang Q, Zheng X, et al. Controllable electrochemical synthesis of Ag nanoparticles in ionic liquid microemulsions. Electrochem. commun. Internet.. 2015;58:41-45. Available from: http://www.sciencedirect.com/science/article/pii/S1388248115001551.

3. Xing $Y$, Jin $Y-Y$, Si J-C, et al. Controllable synthesis and characterization of Fe304/Au composite nanoparticles. J. Magn. Magn. Mater. Internet.. 2014;380:150-156. Available from: http://www.sciencedirect.com/science/article/pii/S0304885314008828.

4. Derakhshandeh PG, Soleimannejad J, Janczak J. Sonochemical synthesis of a new nano-sized cerium(III) coordination polymer and its conversion to nanoceria. Ultrason. Sonochem. Internet.. 2015;26:273-280. Available from: http://linkinghub.elsevier.com/retrieve/pii/S1350417715000358.

5. Alagar M, Theivasant T, Raja a. K. Chemical Synthesis of Nano-sized Particles of Lead Oxide and their Characterization Studies. J. Appl. Sci. 2012;12:398-401.

6. Daou TJ, Pourroy G, Bégin-Colin S, et al. Hydrothermal synthesis of monodisperse magnetite nanoparticles. Chem. Mater. 2006;18:4399-4404.

7. Kandpal N, Sah N, Loshali R. Co-precipitation method of synthesis and characterization of iron oxide nanoparticles. J. Sci. Ind. Res. 2014;73:87-90.

8. Tani T, Mädler L, Pratsinis SE. Homogeneous ZnO nanoparticles by flame spray pyrolysis. J. Nanoparticle Res. 2002;4:337-343.

9. Willard MA, Daniil M, Kniping KE. Nanocrystalline soft magnetic materials at high temperatures: $A$ perspective. Scr. Mater. Internet.. 2012;67:554-559.

10. Polo-Luque ML, Simonet BM, Valc??rcel M. Effect of carbon nanotubes on properties of soft materials based on carbon nanotubes-ionic liquid combinations. Talanta. 2013;110:160-163.

11. Betancourt-Galindo R, Reyes-Rodriguez PY, Puente-Urbina B a., et al. Synthesis of copper nanoparticles by thermal decomposition and their antimicrobial properties. J. Nanomater. 2014;2014:7-11.

12. Cho JS, Hong YJ, Lee J-H, et al. Design and synthesis of micron-sized spherical aggregates composed of hollow Fe2O3 nanospheres for use in lithium-ion batteries. Nanoscale Internet.. 2015.

13. Hayat K, Gondal M a., Khaled MM, et al. Nano ZnO synthesis by modified sol gel method and its application in heterogeneous photocatalytic removal of phenol from water. Appl. Catal. A Gen. Internet.. 2011;393:122-129.

14. Hashemi L, Morsali A. Synthesis and Characterization of a New Nano Lead(II) Two-dimensional Coordination Polymer by Sonochemical Method: A Precursor to Produce Pure Phase Nano-sized Lead(II) Oxide. J. Inorg. Organomet. Polym. Mater. 2010;20:856-861.

15. Zu Z, Hu W, Tang X, et al. A facile method for synthesizing AgInZnS/RGO nanocomposites and their photoelectric detection application. Mater. Lett. Internet.. 2016;182:240-243.

16. Wetchakun N, Chaiwichain S, Wetchakun K, et al. Synthesis and characterization of novel magnetically separable CoFe2O4/CeO2 nanocomposite photocatalysts. Mater. Lett. Internet.. 2013;113:76-79.

17. Mallakpour S, Khadem E. Carbon nanotube-metal oxide nanocomposites: Fabrication, properties and applications Internet.. Chem. Eng. J. 2016.

18. Duval DJ, Risbud SH, Shackelford JF. Ceramic and Glass Materials. Hardcover. 2008.

19. Blair T. Lead oxide technology-Past, present, and future. J. Power Sources. 1998;73:47-55.

20. Wilkinson TJ, Perry DL, Spiller E, et al. A Facile Wet Synthesis of Nanoparticles of Litharge, the Tetragonal Form of PbO. MRS Proc. 2001;704:1-5.

21. Mattesco Patrick, Bui Nam, Simon Patrice AL. passive layer on lead-tin alloys. J. Power Source. 
$1997 ; 64: 21-27$

22. Wang N, Zhou T, Wang J, et al. Sulfide sensor based on the room-temperature phosphorescence of ZnO / SiO 2 nanocomposite †. 2010;82:2386-2393.

23. Karami H, Karimi MA, Haghdar S. Synthesis of uniform nano-structured lead oxide by sonochemical method and its application as cathode and anode of lead-acid batteries. Mater. Res. Bull. 2008;43:3054-3065.

24. Hanifehpour $Y$, Mirtamizdoust B, Farzam AR, et al. Synthesis and Crystal Structure of $\mathrm{Pb}(\mathrm{phen})(\mu-$ N3)( $\mu$-NO3). $n$ and Its Thermal Decomposition to PbO Nanoparticles. J. Inorg. Organomet. Polym. Mater. 2012;22:957-962.

25. Zhou B, Xiao G, Yang X, et al. Pressure-dependent optical behaviors of colloidal CdSe nanoplatelets. Nanoscale Internet.. 2015;7:8835-8842.

26. Ranjbar ZR, Morsali A. Sonochemical syntheses of a new nano-sized porous lead(II) coordination polymer as precursor for preparation of lead(II) oxide nanoparticles. J. Mol. Struct. Internet.. 2009;936:206-212.

27. Shin HJ, Min BK. Direct Synthesis of PbO Nanoparticles from a Lead(II) Nanoflower Coordination Polymer Precursor: Synthesis, Crystal Structure and DFT Calculations of Pb(pcih)N3H2O.n with the Terminal Azide Unit. J. Inorg. Organomet. Polym. Mater. 2013;23:1305-1312.

28. Sonia S, Jayram ND, Suresh Kumar $\mathrm{P}$, et al. Effect of $\mathrm{NaOH}$ concentration on structural, surface and antibacterial activity of $\mathrm{CuO}$ nanorods synthesized by direct sonochemical method. Superlattices Microstruct. Internet.. 2014;66:1-9.

29. Cortez-Valadez M, Vargas-Ortiz a., Rojas-Blanco L, et al. Additional active Raman modes in a-PbO nanoplates. Phys. E Low-Dimensional Syst. Nanostructures Internet.. 2013;53:146-149.

30. Chinnasamy CN, Jeyadevan B, Perales-Perez O, et al. Growth dominant co-precipitation process to achieve high coercivity at room temperature in CoFe/sub 2/O/sub 4/ nanoparticles. IEEE Trans. Magn. 2002;38:2640-2642.

31. Mayekar Jyoti, Dhar Vijay SR. To Study the Role of Temperature and Sodium Hydroxide Concentration in the Synthesis of Zinc. Int. J. Sci. Res. Publ. 2013;3:2-6.

32. Afsin B, Roberts MW. Surface structure and the instability of the formate overlayer at a Pb (110) surface Catal. Lett. 1992:13:277-282.

33. Genc, R, Clergeaud G, Ortiz M, et al. Green Synthesis of Gold Nanoparticles Using GlycerolIncorporated Nanosized Liposomes. Langmuir Acs J. Surfaces Colloids. 2011;27:10894-10900.

34. Pourbafarani S. The Effect of Alkali Concentration on the Structural and Magnetic Properties of MnFerrite Nanoparticles Prepared via the Coprecipitation Method. Metall. Mater. Trans. A Internet.. 2014;45:4535-4537.

35. Taunk PB, Das R, Bisen DP. Synthesis, morphology and structural characterization of lead hydroxide nano particles. Int. J. Res. Appl. Sci. Enginerring Technol. 2014;2:284-288.

36. Maksymiuk K, Stroka J, Galus Z. Chemistry of Lead. Encycl. Electrochem. Power Sources. 2009;762771.

37. Mukhopadhyay I, Raghavan MSS, Sharon M, et al. Photoelectrochemical studies of photoactive lead oxide prepared by the "Potential pulse coupled potentiodynamic anodization technique" in alkaline medium. J. Electroanal. Chem. Internet.. 1994;379:531-534. 